\title{
Metastatic Melanoma Presenting as a Subcutaneous Nodule with Complete Regression of the Primary Lesion
}

\author{
T. G. Liyanage ${ }^{1}$ T. W. Wijesiri ${ }^{1}$, P. Weerasiri ${ }^{2}$
}

\section{INTRODUCTION}

Melanoma is a skin cancer arising from the malignant transformation of melanocytes. Melanoma comprises less than 5\% of malignant skin tumors and it accounts for almost $60 \%$ of lethal skin cancers (1). Skin metastases are not uncommon among patients with melanoma and frequently are the first sign of extra-nodal metastatic disease (2). In 2 to $9 \%$ of the patients presenting with cutaneous melanoma, the primary site of the tumor cannot be detected $(3,4,5,6)$. This can be due to complete regression of the primary lesion.

Here a rare case of metastatic melanoma with complete regression of the primary lesion is described.

\section{CASE REPORT}

A 32-year old woman presented with a gradually enlarging nodule on her lower back. She claimed that there was a small nevus adjacent to the nodule which was there from birth. The nevus gradually enlarged recently with change in colour and regressed with time.

Physical examination revealed a solitary, mobile, soft to firm lump measuring $5 \times 3.5 \times 3.5 \mathrm{~cm}$ which was not attached to the skin. Overlying skin showed a pale blue discoloration without any significant lesions. The initial clinical diagnosis was a lipoma.

Due to the suspicious nature of the lesion during surgery, the lesion was completely excised including the overlying skin. The specimen was submitted for histopathological assessment. Macroscopically there was a fairly circumscribed lesion within the subcutaneous fat. Cut surface revealed a solid brownish black tumour with focal whitish areas. Overlying skin was macroscopically unremarkable.

Microscopical examination revealed a tumour within the subcutaneous fat sparing the dermis and epidermis. The tumour was composed of solid sheets, nests and proliferated fascicles of atypical melanocytes. The cells had nuclei with mild to moderate nuclear pleomorphism with prominent eosinophilic nucleoli. Melanin pigment was seen in the cells with variable melanin distribution. The entire skin ellipse was sampled and examined. There was no evidence of atypical melanocytic proliferation with junctional activity. An area of the dermis resembling the regressed area of the primary lesion was identified. This area showed prominent collections of melanophages associated with fibrosis of the papillary dermis and subepidermal perivascular collections of lymphocytes. Based on the histopathological features, a diagnosis of a metastatic deposit of a malignant melanoma was made. With the absence of skin involvement the usual Clark's or Breslow's staging for melanoma was precluded. The diagnosis was confirmed by positivity for Melan A by immunehistochemistry.

Additional wide local excision was later carried out, including adjacent skin and subcutaneous tissue. It was negative for residual malignancy. As there were no 
LIYANAGE, T. G., WIJESIRI T. W., WEERASIRI P., Metastatic melanoma presenting as a subcutaneous nodule with complete regression of the primary lesion. Sri Lanka Anatomy Journal (SLAJ), 1(2): 38-42, 2017.

other detectable lesions, and no regional lymphadenopathy confirmed by USS, the decision was made for close clinical and radiologic surveillance of the patient.

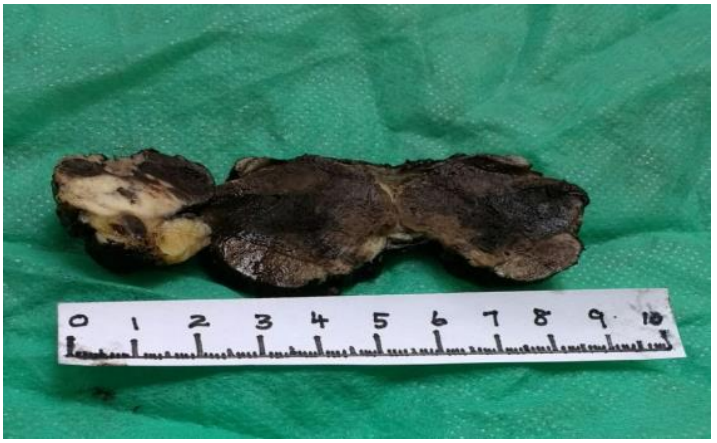

Figure 1: Cut surface of the tumour showing whitish and blackish areas. Black areas represent tumour cells containing melanin pigment.

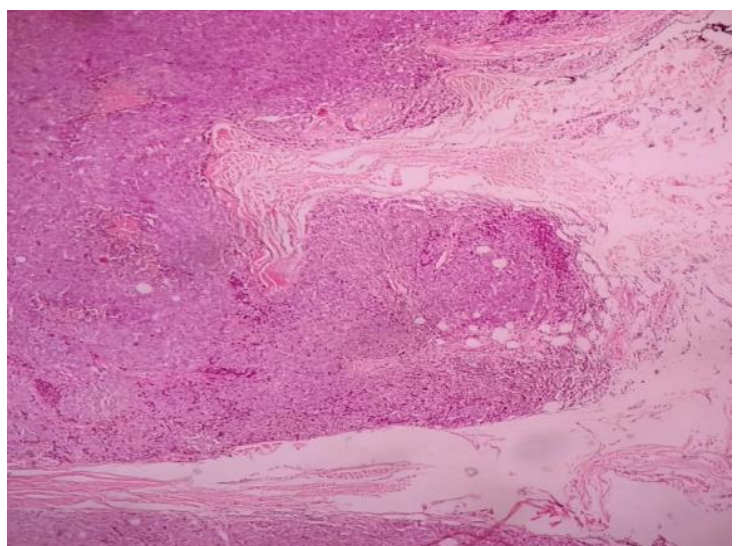

Figure 2: Tumour within the subcutaneous fat (H\&E X100)

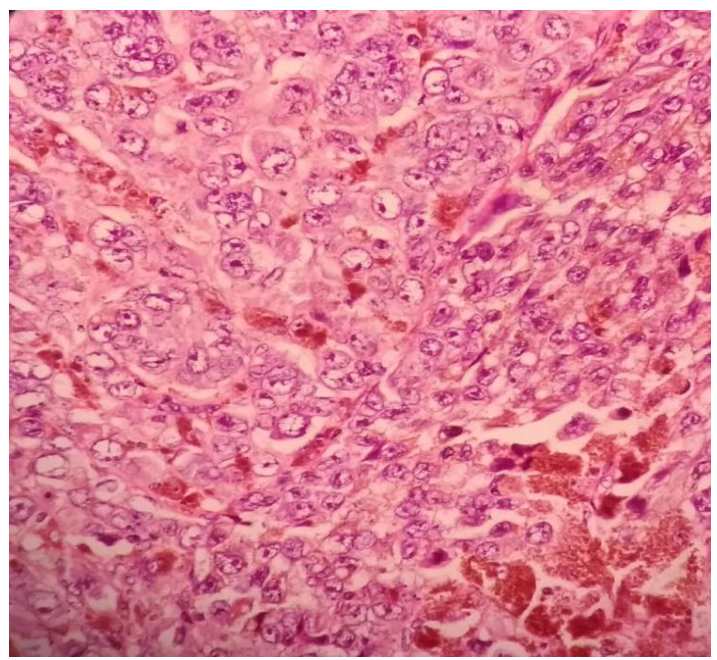

Figure 3: Tumour cells showing prominent eosinophilic nucleoli and intracytoplasmic melanin pigmemt (arrow) (H\&E X400).

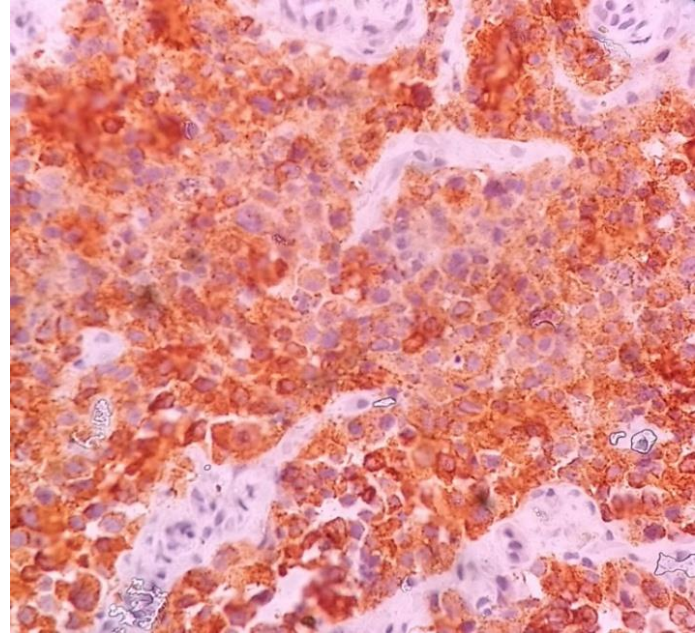

Figure 4: Tumour cells showing strong cytoplasmic Melan A positivity (Immunohistochemistry X400).

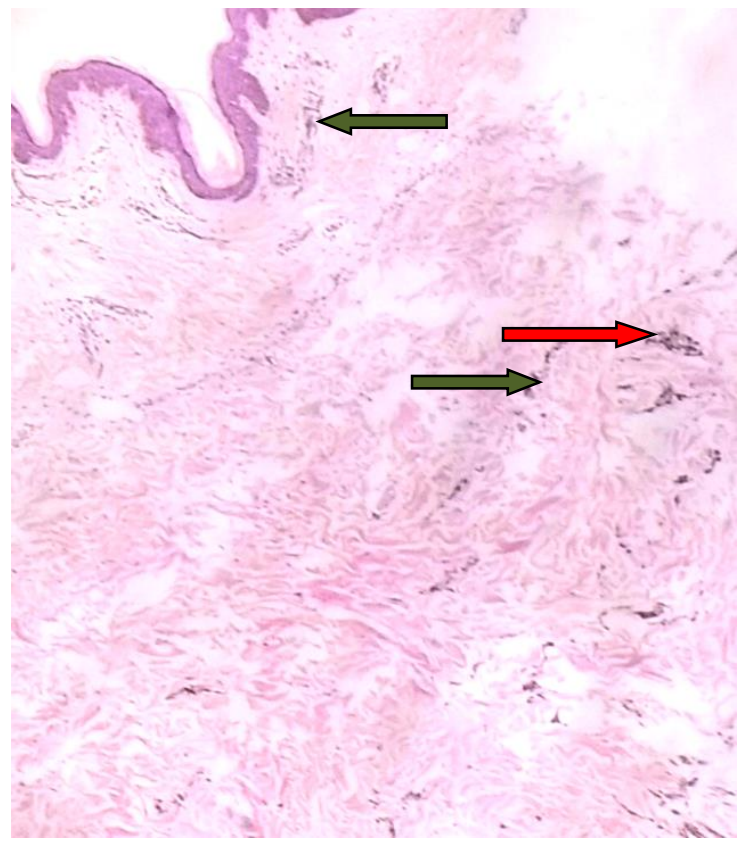

Figure 5: Adjacent dermis showing collections of melanophages (red arrow), perivascular lymphocytes (green arrow) and papillary dermal fibrosis. There are no atypical melanocytes in the epidermis or dermis $(H \& E$ X100)

\section{DISCUSSION}

Melanoma is a lethal skin cancer arising from the malignant transformation of melanocytes. It accounts for $5.2 \%$ all new cancer cases and $1.6 \%$ of all cancer deaths (7). Melanoma is reported as the $19^{\text {th }}$ most common cancer worldwide, with estimated 
LIYANAGE, T. G., WIJESIRI T. W., WEERASIRI P., Metastatic melanoma presenting as a subcutaneous nodule with complete regression of the primary lesion. Sri Lanka Anatomy Journal (SLAJ), 1(2): 38-42, 2017.

age-standardized incidence rates of 2.83.3 per 100,000 (7). Cutaneous melanoma is the most common melanoma subtype, accounting for around $90 \%$ of cases of melanoma (8). The commonest presenting symptom of a melanoma is a pigmented lesion of changing size, followed by a change in colour and pain or tenderness. On clinical examination they usually show papules, nodules or masses in approximately $45 \%$ and as flat topped plaques, macules or patches in approximately $45 \%$ (9). Majority of cutaneous melanomas are associated with sunlight exposure but around $34 \%$ melanomas arise from the malignant transformation within a benign nevoid lesion (9).

Melanomas commonly metastasize to any organ or tissue in the body. Patients with metastatic melanoma generally have a poor prognosis. The duration of survival for metastatic melanoma is less than a year and the median survival is nearly 6 to 8 months (10). Skin metastases are relatively frequent in the natural history of melanoma and can develop in early as well as in late stages of the disease. Around 10$17 \%$ melanoma patients develop cutaneous and subcutaneous metastasis during the disease course (11). Melanoma presenting solely as solitary lesions involving the dermis and/or subcutaneous tissue varies from $0.51 \%$ to $0.92 \%$ (12). In 2 to $9 \%$ of the patients presenting with cutaneous melanoma, the primary site of the tumor cannot be detected $(3,4,5,6)$. This can be explained by complete regression of the primary lesion.

In our patient melanoma presented as a subcutaneous nodule and due to the absence of dermal involvement after extensive sampling of the overlying skin, it was diagnosed to be a secondary deposit from a melanoma. Inspite of thorough clinical examination and radiological evaluation a primary site could not be identified. Considering the patients history, clinical and histopathological evidence, the skin lesion that was present adjacent to the subcutaneous melanoma deposit was assumed as the primary lesion which has regressed completely.

Spontaneous regression of malignant melanoma is defined by the disappearance of melanocytic neoplastic cells partially or completely. In contrast to the partial form, complete regression of primary malignant melanoma is a rare phenomenon, with an incidence of $0.22 \%$ to $0.27 \%$ (13). In our patient also the primary lesion completely regressed leaving the subcutaneous metastatic deposit. Complete regression is commonly seen with metastasis disease and only one case is reported in the literature with complete regression without metastasis (14). Clinically regressing melanoma usually show depigmentation within or around the lesion. Histopathological features of tumour regression include diminishing or disappearing of tumor melanocytes, appearance of newly formed blood vessels, collections of melanophages, lymphocytes in dermis and papillary dermal fibrosis.

The significance of primary regression on the prognosis of melanoma patients is controversial. In early days, studies have demonstrated that melanomas with regression has poor prognosis than melanomas without regression and some studies have failed to demonstrate such a relation (14). According to the current literature, regression in melanoma is less likely to be associated with sentinal lymph node metastasis and therefore now it is regarded as a good prognostic factor (15). However, the incidence of metastatic 
LIYANAGE, T. G., WIJESIRI T. W., WEERASIRI P., Metastatic melanoma presenting as a subcutaneous nodule with complete regression of the primary lesion. Sri Lanka Anatomy Journal (SLAJ), 1(2): 38-42, 2017.

disease in the setting of complete regression of the primary lesion is estimated to range from $4 \%$ to $10 \%$ (16). The regression phenomenon is presumed to be the result of a host immune response. It is proved that natural tumor-specific cytotoxic $\mathrm{T}$ cell response play an important role in primary regressing human melanoma (17).

\section{CONCLUSION}

This case report presents a rare diagnosis of a secondary deposit from a melanoma with complete regression of the primary lesion. The appearance of a solitary subcutaneous metastasis without an evident primary tumor can be explained by spontaneous regression and complete disappearance of the primary melanoma after dissemination to a secondary site. It also should be remembered that in the presence of a melanoma in subcutaneous fat, the clinician needs to perform a meticulous search for a primary lesion and for signs of metastasis in regional lymph nodes and in common metastatic sites. In the setting of localized metastatic disease with complete regression of the primary lesion, it is important of have close clinical and radiological follow up of patients, to identify widely metastatic disease.

\section{REFERENCES}

1. Radovi'c-Kovacevi'c V, Pekmezovi'c T, Adanja B, et al., Survival analysis in patients with cutaneous malignant melanoma. SrpskiArhiv Za Celokupno Lekarstvo. 1997; 125: 132-137. https:// www. ncbi. nlm. nih.gov/pubmed/9265233

2. Lookingbill DP, Spangler N, Helm KF. Cutaneous metastases in patients with metastatic carcinoma: a retrospective study of 4020 patients. J Am Acad Dermatol. 1993; 29: 228-236. https: //www. ncbi.nlm. nih.gov /pubmed/8335743

3. Armado E, Giuliano MD. Clinical aspects of unknown primary melanoma.Ann. Surg.; 1980: 191-198.

4. Milton GW, Lane-Brown MM. Malignant melanoma with an occult primary lesion.

Br. J. Surg. 1967; 54: 651.http:// onlinelibrary. wiley. com/doi/ 10.1002/bjs. 1800540719 /full

5. Milton GW, Shaw HM, McCarthy WH. Occult primary malignant melanoma: factors influencing survival. Br. J. Surg. 1977; $\quad 64: \quad 805$. http://onlinelibrary.wiley.com/doi/10.1002 /bjs.1800641114/full

6. Bulkley GB, Choen MH,Banks PM. Longterm spontaneous regression of malignant melanoma with visceral metastases: Report of a case with immunologic profile. Cancer 1975; 36: 485.

7. Ferlay J, Soerjomataram I, Ervik M, et al.GLOBOCAN 2012, Cancer Incidence and Mortality Worldwide: IARC CancerBase No. 11 [Internet].Lyon, France: International Agency for Research on Cancer; 2013. Available from:http:// globocan.iarc.fr, accessed on 21/08/2017.

8. Chang AE, Karnell LH, Menck HR. The National Cancer Data Base report on cutaneous and noncutaneous melanoma: a summary of 84,836 cases from the past decade. The American College of Surgeons Commission on Cancer and the American Cancer Society. Cancer 1998; 83:1664-1678.

https://www.ncbi.nlm.nih.gov/pubmed/97 $\underline{81962}$

9. Hui SK, Lau KH, Ó CYL. Original Article Cutaneous melanoma: clinical features of 32 Hong Kong Chinese patients. Hong Kong J. Dermatol. Venereol.2005; 13: $130-40$.

10. Tas F. Metastatic behavior in melanoma: Timing, pattern, survival, and influencing Factors. 2012; 2012. https://www. hindawi.com /journals /jo/2012/647684/

11. Lookingbill DP, Spangler N, Helm KF. Cutaneous metastases in patients with metastatic carcinoma: a retrospective study of 4020 patients. J Am Acad Dermatol. $\quad 1993 ; \quad 29: \quad 228-236$. https://www.ncbi.nlm.nih.Gov /pubmed $\underline{18335743}$ 
LIYANAGE, T. G., WIJESIRI T. W., WEERASIRI P., Metastatic melanoma presenting as a subcutaneous nodule with complete regression of the primary lesion. Sri Lanka Anatomy Journal (SLAJ), 1(2): 38-42, 2017.

12. Bowen GM, Chang AE, Lowe L, Hamilton T. Solitary Melanoma Confined to the Dermal and/or Subcutaneous Tissue. 2000; 136: 8-10.

13. Emanue PO, Mannion M, Phelps RG. Complete regression of primary malignant melanoma. Am J Dermatopathol.2008; 30(2):178-181. http:// www. rjme.ro/RJME/ resources/ files/ 551 214635642.pdf

14. Requena C, Botella-estrada R, Traves V, Nagore E, Almenar S, Guillén C. Problems in Defining Melanoma Regression and Prognostic Implication. 2009; 759-66. https://www. ncbi. nlm. nih.gov/pubmed/19889297

15. Ribero S, Moscarella E, Ferrara G, et al. Regression in cutaneous melanoma: a comprehensive review from diagnosis to prognosis. J. Eur. Acad. Dermatology Venereol. 2016; 30: 2030-2037. https://www.ncbi.nlm.nih. gov/ pubmed/27401335

16. High WA, Stewart D, Wilbers CR, et al. Completely regressed primary cutaneous malignant melanoma with nodal and/or visceral metastases:a report of 5 casles and assessment of the literature and diagnostic criteria. J Am AcadDermatol.2005; 53: 89-100.

Available from: https://www.ncbi.nlm. Nih. gov/pubmed/15965428

17. 17. Zorn E, Hercend T. A natural cytotoxic $\mathrm{T}$ cell response in a spontaneously regressing human melanoma targets a neoantigen resulting from a somatic point mutation. Eur $\mathbf{J}$ Immunol.1999; 29(2): 592-601.

Available from: https://www.ncbi.nlm.nih.gov/pubmed/10 064075

\section{CORRESPONDENCE}

Dr. T. G. Liyanage

Department of Pathology, University of Ruhuna

E mail-thusharieliyanage@yahoo.com

Received: September 2017

Accepted: September 2017 